\title{
Super compact equation for water waves
}

\author{
A. I. Dyachenko ${ }^{1,2} \dagger$, D. I. Kachulin ${ }^{2}$ and V. E. Zakharov ${ }^{1,2,3,4,5}$ \\ ${ }^{1}$ Landau Institute for Theoretical Physics, 142432, Chernogolovka, Russia \\ ${ }^{2}$ Novosibirsk State University, 630090, Novosibirsk-90, Russia \\ ${ }^{3}$ Department of Mathematics, University of Arizona, Tucson, AZ, 857201, USA \\ ${ }^{4}$ Physical Institute of RAS, Leninskiy prospekt, 53, Moscow, 119991, Russia \\ ${ }^{5}$ Space Research Institute of RAS, 84/32 Profsoyuznaya Str, Moscow, 117997, Russia
}

(Received xx; revised xx; accepted $\mathrm{xx}$ )

Mathematicians and physicists have long been interested in the subject of water waves. The problems formulated in this subject can be considered fundamental, but many questions remain unanswered. For instance, a satisfactory analytic theory of such a common and important phenomenon as wave breaking has yet to be developed. Our knowledge of the formation of rogue waves is also fairly poor despite the many efforts devoted to this subject.

One of the most important tasks of the theory of water waves is the construction of simplified mathematical models that are applicable to the description of these complex events under the assumption of weak nonlinearity. The Zakharov equation, as well as the Nonlinear Schrödinger equation (NLSE) and the Dysthe equation (which are actually its simplifications), are among them.

In this article, we derive a new modification of the Zakharov equation based on the assumption of unidirectionality (the assumption that all waves propagate in the same direction). To derive the new equation, we use the Hamiltonian form of the Euler equation for an ideal fluid and perform a very specific canonical transformation. This transformation is possible due to the "miraculous" cancellation of the nontrivial fourwave resonant interaction in the one-dimensional wave field. The obtained equation is remarkably simple. We call the equation the "super compact water wave equation". This equation includes a nonlinear wave term (à la NLSE) together with an advection term that can describe the initial stage of wave-breaking. The NLSE and the Dysthe equations (Dysthe (1979)) can be easily derived from the super compact equation. This equation is also suitable for analytical studies as well as for numerical simulation. Moreover, this equation also allows one to derive a spatial version of the water wave equation that describes experiments in flumes and canals.

\section{Introduction}

A potential flow of an ideal incompressible fluid with a free surface in a gravity field is described (Zakharov 1968) by the following Hamiltonian system:

$$
\frac{\partial \psi}{\partial t}=-\frac{\delta H}{\delta \eta} \quad \frac{\partial \eta}{\partial t}=\frac{\delta H}{\delta \psi} .
$$

Hereafter, we study only the case of one horizontal direction: unidirectional waves. Now,

$$
\begin{aligned}
& \eta=\eta(x, t) \text { - shape of the surface, } \\
& \psi=\psi(x, t)=\phi(x, \eta(x, t), t) \text { - potential on the surface, }
\end{aligned}
$$

$\dagger$ Email address for correspondence: alexd@itp.ac.ru 


$$
\phi(x, z, t) \text { - potential inside the fluid. }
$$

The Hamiltonian $H$ is

$$
H=\frac{1}{2} \int d x \int_{-\infty}^{\eta}|\nabla \phi|^{2} d z+\frac{g}{2} \int \eta^{2} d x
$$

The potential $\phi(x, z, t)$ satisfies the Laplace equation:

$$
\frac{\partial^{2} \phi}{\partial x^{2}}+\frac{\partial^{2} \phi}{\partial z^{2}}=0
$$

with the asymptotic boundary conditions:

$$
\frac{\partial \phi}{\partial z} \rightarrow 0, \quad \text { at } z \rightarrow-\infty
$$

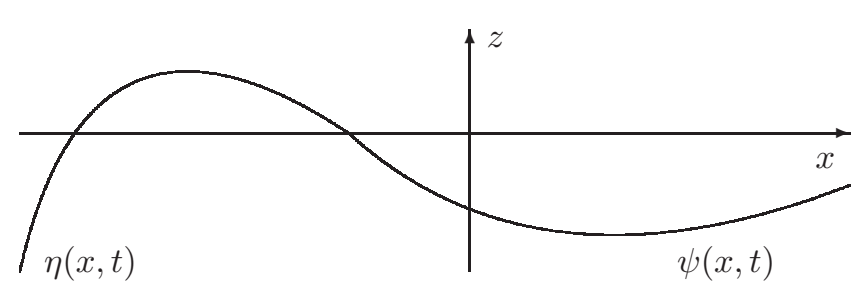

If the steepness of the surface is small, $\left|\eta_{x}\right|<<1$, the Hamiltonian can be represented by the infinite series

$$
\begin{aligned}
H & =H_{2}+H_{3}+H_{4}+\ldots \\
H_{2} & =\frac{1}{2} \int\left(g \eta^{2}+\psi \hat{k} \psi\right) d x, \\
H_{3} & =-\frac{1}{2} \int\left\{(\hat{k} \psi)^{2}-\left(\psi_{x}\right)^{2}\right\} \eta d x, \\
H_{4} & =\frac{1}{2} \int\left\{\psi_{x x} \eta^{2} \hat{k} \psi+\psi \hat{k}(\eta \hat{k}(\eta \hat{k} \psi))\right\} d x
\end{aligned}
$$

where $\hat{k} \psi$ means multiplication by $|k|$ in $k$-space $\left(\hat{k}=\sqrt{-\frac{\partial^{2}}{\partial x^{2}}}\right)$.

Equations (1.1), although truncated according to (1.4), even for the full 3-D case, can be efficiently used for numerical simulations of water wave dynamics (see, for instance, (Korotkevich et al 2008)). However, they are not convenient for analytic study because $\eta(x, t)$ and $\psi(x, t)$ are not "optimal" canonical variables. One can choose better Hamiltonian variables by performing a proper canonical transformation. This transformation can be achieved in two steps. In the first step, we eliminate all third-order terms and some fourth-order terms - all so-called "nonresonant" cubic and quartic terms in the Hamiltonian. What we obtain as a result of this transformation is the so-called Zakharov equation, which has been widely used in recent years by many researchers (see, for instance, (Crawford et al 1980; Debnath 1994)) and more recent publications (Annenkov \& Shrira 2011, 2013). In the second step, one can "improve" the Zakharov equation by applying an appropriate canonical transformation to simplify the only remaining resonant fourth-order term. This "improvement" is possible due to a very special property of the quartic Hamiltonian in the Zakharov equation, specifically, an unexpected cancellation (Dyachenko \& Zakharov 1994) of nontrivial four-wave interactions. This cancellation only occurs in the one-dimensional case and makes it possible to replace the "generic" Zakharov equation by a substantially more suitable "compact equation", (Dyachenko \& Zakharov 2012), which was intensively used as a base for both numerical simulations 
(Fedele \& Dutykh 2012a,b; Dyachenko 2013; Dyachenko et al 2014; Fedele 2014a,b; Dyachenko et al 2015,a,b) and an analytical proof of the nonintegrability of the Zakharov equation (Dyachenko et al 2013a).

In this paper, we analyzed this second step in the canonical transformation, which is not a unique procedure. One can accomplish this in many different ways, thereby obtaining different forms of the compact equation. Here, we present the most optimal (in our opinion) version of the compact equation, which we call "the super compact equation" for water waves. In addition, we present some preliminary results of the numerical simulations of the super compact equation.

It should be mentioned that this new equation enables a remarkably straightforward derivation of the spatial version of the equation. The spatial compact equation solves the Cauchy problem in space and is an exceptionally convenient tool for comparison of the theory and experimental study in laboratory flumes for nonlinear gravity waves (Dyachenko \& Zakharov (2016)).

\section{The Zakharov equation}

For a detailed derivation of the Zakharov equation, see references (Zakharov (1968); Krasitskii (1990); Zakharov et al (1992)). A brief outline starting with the Hamiltonian (1.4) is given as follows:

1. We introduce complex normal variables $a_{k}$ :

$$
\eta_{k}=\sqrt{\frac{\omega_{k}}{2 g}}\left(a_{k}+a_{-k}^{*}\right) \quad \psi_{k}=-i \sqrt{\frac{g}{2 \omega_{k}}}\left(a_{k}-a_{-k}^{*}\right)
$$

Here, $\omega_{k}=\sqrt{g|k|}$ is the dispersion law for the gravity waves, and the Fourier transformations $\psi(x) \rightarrow \psi_{k}$ and $\eta(x) \rightarrow \eta_{k}$ are defined as follows:

$$
f_{k}=\frac{1}{\sqrt{2 \pi}} \int f(x) e^{-i k x} d x, \quad f(x)=\frac{1}{\sqrt{2 \pi}} \int f_{k} e^{+i k x} d k .
$$

In the new variables $a_{k}$, the Hamiltonian takes the following form:

$$
\begin{aligned}
H_{2} & =\int \omega_{k} a_{k} a_{k}^{*} d k \\
H_{3} & =\int V_{k_{1} k_{2}}^{k}\left\{a_{k}^{*} a_{k_{1}} a_{k_{2}}+a_{k} a_{k_{1}}^{*} a_{k_{2}}^{*}\right\} \delta_{k-k_{1}-k_{2}} d k d k_{1} d k_{2} \\
& +\frac{1}{3} \int U_{k k_{1} k_{2}}\left\{a_{k} a_{k_{1}} a_{k_{2}}+a_{k}^{*} a_{k_{1}}^{*} a_{k_{2}}^{*}\right\} \delta_{k+k_{1}+k_{2}} d k d k_{1} d k_{2}, \\
H_{4} & =\frac{1}{2} \int W_{k_{1} k_{2}}^{k_{3} k_{4}} a_{k_{1}}^{*} a_{k_{2}}^{*} a_{k_{3}} a_{k_{4}} \delta_{k_{1}+k_{2}-k_{3}-k_{4}} d k_{1} d k_{2} d k_{3} d k_{4}+ \\
& +\frac{1}{3} \int G_{k_{1} k_{2} k_{3}}^{k_{4}}\left(a_{k_{1}}^{*} a_{k_{2}}^{*} a_{k_{3}}^{*} a_{k_{4}}+\text { c.c. }\right) \delta_{k_{1}+k_{2}+k_{3}-k_{4}} d k_{1} d k_{2} d k_{3} d k_{4}+ \\
& +\frac{1}{12} \int R_{k_{1} k_{2} k_{3} k_{4}}\left(a_{k_{1}}^{*} a_{k_{2}}^{*} a_{k_{3}}^{*} a_{k_{4}}^{*}+\text { c.c. }\right) \delta_{k_{1}+k_{2}+k_{3}+k_{4}} d k_{1} d k_{2} d k_{3} d k_{4}
\end{aligned}
$$

For our purposes, the exact expressions for the coefficients of the Hamiltonian are unimportant. Nevertheless, a careful reader can find them in references (Zakharov (1998, 1999); Dyachenko et al (2015)).

The equations of motion (1.1) now yield the following:

$$
\frac{\partial a_{k}}{\partial t}+i \frac{\delta H}{\delta a_{k}^{*}}=0
$$


2. In the variables $a_{k}$, the Hamiltonian contains nonresonant three-wave interactions, and hence, the variables are still suboptimal. We introduce yet another set of variables $b_{k}$ by another canonical transformation $a_{k} \rightarrow b_{k}$ to cancel all the nonresonant cubic and quartic terms in the new Hamiltonian. An efficient way to construct this transformation was offered in reference (Zakharov et al 1992) and can be written as follows:

$$
\begin{gathered}
a_{k}=b_{k}+\int\left[2 \tilde{V}_{k k_{2}}^{k_{1}} b_{k_{1}} b_{k_{2}}^{*} \delta_{k_{1}-k-k_{2}}-\tilde{V}_{k_{1} k_{2}}^{k} b_{k_{1}} b_{k_{2}} \delta_{k-k_{1}-k_{2}}-\tilde{U}_{k k_{1} k_{2}} b_{k_{1}}^{*} b_{k_{2}}^{*} \delta_{k+k_{1}+k_{2}}\right] d k_{1} d k_{2} \\
+\int\left[A_{k_{1} k_{2} k_{3}}^{k} b_{k_{1}} b_{k_{2}} b_{k_{3}}+A_{k_{2} k_{3}}^{k k_{1}} b_{k_{1}}^{*} b_{k_{2}} b_{k_{3}}+A_{k_{3}}^{k k_{1} k_{2}} b_{k_{1}}^{*} b_{k_{2}}^{*} b_{k_{3}}+A^{k k_{1} k_{2} k_{3}} b_{k_{1}}^{*} b_{k_{2}}^{*} b_{k_{3}}^{*}\right] d k_{1} d k_{2} d k_{3},
\end{gathered}
$$

where the exact expressions for the coefficients of the transformation (2.1) can be found in Dyachenko et al (2015). The resulting Hamiltonian after the transformation yields

$$
H=\int \omega_{k} b_{k} b_{k}^{*} d k+\frac{1}{2} \int T_{k k_{1}}^{k_{2} k_{3}} b_{k}^{*} b_{k_{1}}^{*} b_{k_{2}} b_{k_{3}} \delta_{k+k_{1}-k_{2}-k_{3}} d k d k_{1} d k_{2} d k_{3}+\tilde{H},
$$

where $\tilde{H}$ is an infinite series in $b_{k}, b_{k}^{*}$ starting from the fifth-order terms. The explicit (and cumbersome) expression for $T_{k k_{1}}^{k_{2} k_{3}}$ can be found in (Zakharov 1968; Zakharov 1998, 1999). The motion equation

$$
\frac{\partial b_{k}}{\partial t}+i \frac{\delta H}{\delta b_{k}^{*}}=0
$$

(neglecting $\tilde{H}$ ) is the traditional Zakharov equation.

\section{Canonical transformation for the Zakharov equation}

A possibility for further simplification of equation (2.3) is based on the following remarkable fact, established in (Dyachenko \& Zakharov 1994). Let us consider the resonance conditions for the four-wave interactions:

$$
\begin{aligned}
k+k_{1} & =k_{2}+k_{3}, \\
\omega_{k}+\omega_{k_{1}} & =\omega_{k_{2}}+\omega_{k_{3}},
\end{aligned}
$$

In the 1-D case, all solutions of this system of equations (3.1) can be divided into two parts - so-called "trivial" and "nontrivial" parts. The "nontrivial" solution can be solved as follows:

$$
\begin{aligned}
k & =a(1+\zeta)^{2}, \\
k_{1} & =a(1+\zeta)^{2} \zeta^{2}, \\
k_{2} & =-a \zeta^{2}, \\
k_{3} & =a\left(1+\zeta+\zeta^{2}\right)^{2} \quad \text { here } 0<\zeta<1 .
\end{aligned}
$$

Notice the product $k k_{1} k_{2} k_{3}<0$. Now,

$$
T_{k k_{1}}^{k_{2} k_{3}}=F(a, \zeta)=a^{3} f(\zeta) .
$$

Direct calculation shows that for the "nontrivial" resonance (3.2),

$$
f(\zeta) \equiv 0 .
$$

This fact means that "nontrivial" four-wave resonances are absent! 
Moreover, $T_{k k_{1}}^{k_{2} k_{3}} \equiv 0$ if the product $k k_{1} k_{2} k_{3} \leqslant 0$. In addition, it has a very simple form:

$$
T_{k_{2} k_{3}}^{k k_{1}}=\theta\left(k k_{1} k_{2} k_{3}\right) \frac{\left(k k_{1} k_{2} k_{3}\right)^{\frac{1}{2}}}{4 \pi}\left[\left(\frac{\omega \omega_{1}}{\omega_{2} \omega_{3}}\right)^{\frac{1}{2}}+\left(\frac{\omega_{2} \omega_{3}}{\omega \omega_{1}}\right)^{\frac{1}{2}}\right] \min \left(k, k_{1}, k_{2}, k_{3}\right)
$$

$\min \left(k, k_{1}, k_{2}, k_{3}\right) \quad$ is minimum of $\left(k, k_{1}, k_{2}, k_{3}\right)$.

Here, $\theta(k)$ is the step function

$$
\theta(x)= \begin{cases}0 & \text { if } x \leqslant 0 \\ 1 & \text { if } x>0\end{cases}
$$

Obviously, for positive $k_{i}$ with resonant condition

$$
\begin{gathered}
k+k_{1}=k_{2}+k_{3}, \\
\min \left(k, k_{1}, k_{2}, k_{3}\right)=\frac{1}{4}\left(k+k_{1}+k_{2}+k_{3}-\left|k-k_{2}\right|-\left|k-k_{3}\right|-\left|k_{1}-k_{2}\right|-\left|k_{1}-k_{3}\right|\right) .
\end{gathered}
$$

This means that a system initially consisting of unidirectional waves retains this property for all times. Indeed, a wave with negative $k$ can appear only from the following equation with all positive $k$ in the R.H.S.:

$$
i \frac{\partial b_{k}}{\partial t}=\omega_{k} b_{k}+\int T_{k k_{1}}^{k_{2} k_{3}} b_{k_{1}}^{*} b_{k_{2}} b_{k_{3}} \delta_{k+k_{1}-k_{2}-k_{3}} d k_{1} d k_{2} d k_{3}
$$

However, $T_{k k_{1}}^{k_{2} k_{3}}$ for such a selection of $k$ is identically zero.

Now, one can see that the Zakharov equation is a compact equation but can be further "improved". In other words, the coefficient (3.4) can be simplified even further.

The system (3.1) also has the following "trivial" solution:

$$
k_{2}=k_{1}, \quad k_{3}=k, \quad \text { or } \quad k_{2}=k, \quad k_{3}=k_{1} .
$$

We introduce $T_{k k_{1}}$ (diagonal part) as the value of the four-wave coefficient on the trivial manifold (3.6). This was calculated in (Zakharov 1968) and is equal to

$$
T_{k k_{1}}=T_{k k_{1}}^{k k_{1}}=\frac{1}{4 \pi}|k|\left|k_{1}\right|\left(\left|k+k_{1}\right|-\left|k-k_{1}\right|\right)=\frac{1}{2 \pi}|k|\left|k_{1}\right| \min \left(|k|,\left|k_{1}\right|\right)
$$

Let us introduce $\tilde{T}_{k k_{1}}^{k_{2} k_{3}}$ as follows:

$$
\tilde{T}_{k k_{1}}^{k_{2} k_{3}}=\theta\left(k k_{1} k_{2} k_{3}\right)\left[\frac{1}{2}\left(T_{k k_{2}}+T_{k k_{3}}+T_{k_{1} k_{2}}+T_{k_{1} k_{3}}\right)-\frac{1}{4}\left(T_{k k}+T_{k_{1} k_{1}}+T_{k_{2} k_{2}}+T_{k_{3} k_{3}}\right)\right]
$$

A canonical transformation of the second step replaces Zakharov's $T_{k k_{1}}^{k_{2} k_{3}}$ from (2.2) with the simpler $\tilde{T}_{k k_{1}}^{k_{2} k_{3}}$ while keeping their diagonal part the same.

The simple method to construct the canonical transformation is based on the fact that a Hamiltonian system (with variable $\tilde{c}_{k}(t)$ ) is invariant under translation in time and that the transformation $\tilde{c}_{k}(0) \rightarrow \tilde{c}_{k}(\tau)$ is canonical. Let us construct this transformation (as a power series) using an auxiliary Hamiltonian $\tilde{\mathcal{H}}$ (starting from the quartic term) of the form:

$$
\tilde{\mathcal{H}}=\frac{1}{2} \int \tilde{\mathbf{B}}_{\mathbf{k k}_{1}}^{\mathbf{k}_{2} \mathbf{k}_{3}} \tilde{c}_{k}^{*} \tilde{c}_{k_{1}}^{*} \tilde{c}_{k_{2}} d_{k_{3}} \delta_{k+k_{1}-k_{2}-k_{3}} d k d k_{1} d k_{2} d k_{3}+\ldots
$$

where the symmetry relations

$$
\tilde{\mathbf{B}}_{\mathbf{k k}_{1}}^{\mathbf{k}_{2} \mathbf{k}_{3}}=\tilde{\mathbf{B}}_{\mathbf{k}_{1} \mathbf{k}}^{\mathbf{k}_{2} \mathbf{k}_{3}}=\tilde{\mathbf{B}}_{\mathbf{k k}_{1}}^{\mathbf{k}_{3} \mathbf{k}_{2}}=\left(\tilde{\mathbf{B}}_{\mathbf{k}_{2} \mathbf{k}_{3}}^{\mathbf{k} \mathbf{k}_{1}}\right)^{*}
$$


are necessary to obtain a real-valued $\tilde{\mathcal{H}}$. Using a Taylor series, we can express the old canonical $b_{k}(\tau)=\tilde{c}_{k}(\tau)$ in terms of $\tilde{c}_{k}(0)$ :

$$
\begin{aligned}
\tilde{c}_{k}(\tau) & =\tilde{c}_{k}(0)+\left.\tau \frac{\partial \tilde{c}_{k}(\tau)}{\partial \tau}\right|_{\tau=0}+\ldots \\
\left.\frac{\partial \tilde{c}_{k}(\tau)}{\partial \tau}\right|_{\tau=0} & =-\left.i \frac{\delta \tilde{\mathcal{H}}\left(\tilde{c}_{k}(\tau), \tilde{c}_{k}^{*}(\tau)\right)}{\delta \tilde{c}_{k}^{*}(\tau)}\right|_{\tau=0}
\end{aligned}
$$

and

$$
b_{k}=\tilde{c}_{k}-i \int \tilde{\mathbf{B}}_{\mathbf{k k}_{1}}^{\mathbf{k}_{2} \mathbf{k}_{3}} \tilde{c}_{k_{1}}^{*} \tilde{c}_{k_{2}} \tilde{c}_{k_{3}} \delta_{k+k_{1}-k_{2}-k_{3}} d k_{1} d k_{2} d k_{3}+\ldots
$$

is a canonical transformation. Now, we plug this transformation into the Hamiltonian (2.2) of the Zakharov equation and obtain the new Hamiltonian:

$$
\begin{aligned}
H=\int \omega_{k} \tilde{c}_{k} \tilde{c}_{k}^{*} d k & +\frac{1}{2} \iint_{k}\left[T_{k k_{1}}^{k_{2} k_{3}}-i\left(\omega_{k}+\omega_{k_{1}}-\omega_{k_{2}}-\omega_{k_{3}}\right) \tilde{\mathbf{B}}_{\mathbf{k k}_{1}}^{\mathbf{k}_{2} \mathbf{k}_{3}}\right] \times \\
& \times \tilde{c}_{k}^{*} \tilde{c}_{k_{1}}^{*} \tilde{c}_{k_{2}} d_{k_{3}} \delta_{k+k_{1}-k_{2}-k_{3}} d k d k_{1} d k_{2} d k_{3}+\ldots
\end{aligned}
$$

The coefficient $\tilde{\mathbf{B}}_{\mathbf{k k}_{1}}^{\mathbf{k}_{\mathbf{2}} \mathbf{k}_{\mathbf{3}}}$ of the auxiliary Hamiltonian is the same as the coefficient of the canonical transformation. It controls the four-wave coefficient $T_{k k_{1}}^{k_{2} k_{3}}$ in the Hamiltonian of the Zakharov equation (3.10). To replace Zakharov's $T_{k k_{1}}^{k_{2} k_{3}}$ by the simpler $\tilde{T}_{k k_{1}}^{k_{2} k_{3}}$, the coefficient $\tilde{\mathbf{B}}_{\mathbf{k k}_{\mathbf{1}}}^{\mathbf{k}_{\mathbf{2}} \mathbf{k}_{\mathbf{3}}}$ has to be equal to

$$
\tilde{\mathbf{B}}_{\mathbf{k k}_{1}}^{\mathbf{k}_{2} \mathbf{k}_{3}}=i \frac{\tilde{T}_{k k_{1}}^{k_{2} k_{3}}-T_{k k_{1}}^{k_{2} k_{3}}}{\omega_{k}+\omega_{k_{1}}-\omega_{k_{2}}-\omega_{k_{3}}} .
$$

One can check that $\tilde{\mathbf{B}}_{\mathbf{k k}_{1}}^{\mathbf{k}_{2} \mathbf{k}_{3}}$ has no singularities at $k+k_{1}=k_{2}+k_{3}$. Indeed, in the region where the product $k k_{1} k_{2} k_{3} \leqslant 0$, the singularities are canceled by virtue of the identity (3.3). In the region where the product $k k_{1} k_{2} k_{3}>0$, the singularities are canceled due to the special choice of $\tilde{T}_{k k_{1}}^{k_{2} k_{3}}$. The exact expression for $\tilde{\mathbf{B}}_{\mathbf{k k}_{1}}^{\mathbf{k}_{2} \mathbf{k}_{\mathbf{3}}}$ was published in (Dyachenko et al 1995). This leads to the derivation of the "compact water wave equation" (not yet the super compact).

Due to the absence of nontrivial resonances, waves moving in the same direction do not generate waves moving in the opposite direction, and hence, we can assume without loss of generality that for all wavenumbers $k_{i}>0$, this leads to the following simplification:

$$
\begin{aligned}
\tilde{T}_{k k_{1}}^{k_{2} k_{3}} & =\left[-\frac{1}{8 \pi}\left(k k_{2}\left|k-k_{2}\right|+k k_{3}\left|k-k_{3}\right|+k_{1} k_{2}\left|k_{1}-k_{2}\right|+k_{1} k_{3}\left|k_{1}-k_{3}\right|\right)+\right. \\
& \left.+\frac{1}{8 \pi}\left(k k_{1}\left(k+k_{1}\right)+k_{2} k_{3}\left(k_{2}+k_{3}\right)\right)\right] \theta(k) \theta\left(k_{1}\right) \theta\left(k_{2}\right) \theta\left(k_{3}\right)
\end{aligned}
$$

Returning from the Fourier space, we can write the following compact expression for the Hamiltonian in $x$-space:

$$
H=\int \tilde{c}^{*} \hat{\omega} \tilde{c} d x+\frac{1}{2} \int\left|\frac{\partial \tilde{c}}{\partial x}\right|^{2}\left[\frac{i}{2}\left(\tilde{c} \frac{\partial \tilde{c}^{*}}{\partial x}-\tilde{c}^{*} \frac{\partial \tilde{c}}{\partial x}\right)-\hat{k}|\tilde{c}|^{2}\right] d x
$$

where $\hat{\omega}$ denotes multiplication by $\sqrt{g|k|}$ in Fourier space. The compact equation, with Hamiltonian (3.13), was used for the numerical simulations in papers (Dyachenko \& Zakharov 2012; Fedele \& Dutykh 2012a,b). 


\section{Super compact equation}

Note that the choice of (3.7) is not unique for introducing the new Hamiltonian. The conditions imposed on $\tilde{T}_{k k_{1}}^{k_{2} k_{3}}$ are rather loose:

(i) The symmetry conditions require that

$$
\tilde{\mathbf{T}}_{\mathbf{k k}_{1}}^{\mathbf{k}_{2} \mathbf{k}_{3}}=\tilde{\mathbf{T}}_{\mathbf{k}_{1} \mathbf{k}}^{\mathbf{k}_{2} \mathbf{k}_{3}}=\tilde{\mathbf{T}}_{\mathbf{k k}_{1}}^{\mathbf{k}_{3} \mathbf{k}_{2}}=\tilde{\mathbf{T}}_{\mathbf{k}_{2} \mathbf{k}_{3}}^{\mathrm{k}_{\mathbf{k}_{1}}}
$$

(ii) The diagonal part must be strictly defined as

$$
\tilde{\mathbf{T}}_{\mathbf{k k}_{\mathbf{1}}}^{\mathbf{k}_{2} \mathbf{k}_{3}}=T_{k k_{1}}=\frac{1}{4 \pi}|k|\left|k_{1}\right|\left(\left|k+k_{1}\right|-\left|k-k_{1}\right|\right)=\frac{1}{2 \pi}|k|\left|k_{1}\right| \min \left(|k|,\left|k_{1}\right|\right) .
$$

The symmetry conditions suggest that $\tilde{\mathbf{T}}_{\mathbf{k k}_{1}}^{\mathbf{k}_{2} \mathbf{k}_{3}}$ may be invariant under permutations of all $k_{i}$. Let us choose $\tilde{\mathbf{T}}_{\mathbf{k k}_{1}}^{\mathbf{k}_{2} \mathbf{k}_{3}}$ as follows:

$$
\begin{aligned}
\tilde{\mathbf{T}}_{\mathbf{k k}_{1}}^{\mathbf{k}_{2} \mathbf{k}_{3}} & =\frac{\left(k k_{1} k_{2} k_{3}\right)^{\frac{1}{2}}}{2 \pi} \min \left(k, k_{1}, k_{2}, k_{3}\right) \theta_{k} \theta_{k_{1}} \theta_{k_{2}} \theta_{k_{3}}, \\
\theta_{k} & - \text { is the step function, } \theta_{k}=\theta(k)
\end{aligned}
$$

and $\min \left(k, k_{1}, k_{2}, k_{3}\right)$ is defined in (3.4) and (3.5). We substitute the new $\tilde{\mathbf{T}}_{\mathbf{k k}_{\mathbf{1}}}^{\mathbf{k}_{\mathbf{2}} \mathbf{k}_{\mathbf{3}}}$ (4.1) into the coefficient $\tilde{\mathbf{B}}_{\mathbf{k k}_{\mathbf{1}}}^{\mathbf{k}_{\mathbf{2}} \mathbf{k}_{\mathbf{3}}}$ (3.11) and apply the canonical transformation (3.9). After that transformation, the functions $\tilde{c}_{k}$ satisfy the following equation:

$$
\begin{aligned}
& i \dot{\tilde{c_{k}}}=\frac{\delta H}{\delta \tilde{c}_{k}^{*}}=\omega_{k} \tilde{c}_{k}+ \\
& +\frac{k^{\frac{1}{2}} \theta_{k}}{2 \pi} \int \min \left(k, k_{1}, k_{2}, k_{3}\right)\left(k_{1}^{\frac{1}{2}} \theta_{k_{1}} \tilde{c}_{k_{1}}^{*}\right)\left(k_{2}^{\frac{1}{2}} \theta_{k_{2}} \tilde{c}_{k_{2}}\right)\left(k_{3}^{\frac{1}{2}} \theta_{k_{3}} \tilde{c}_{k_{3}}\right) \delta_{k+k_{1}-k_{2}-k_{3}} d k_{1} d k_{2} d k_{3}(4
\end{aligned}
$$

It is convenient to introduce a new Hamiltonian variable:

$$
c_{k}=k^{\frac{1}{2}} \theta_{k} \tilde{c}_{k} .
$$

$c_{k}$ is the Fourier transform of a function analytic in the upper complex half-plane. Note, the nonlinear term in (4.2) preserves the analyticity property. Multiplying (4.2) by $i k^{\frac{1}{2}}$ and using the definition of $c_{k}$ (4.3) results in

$$
\dot{c}_{k}+i k \theta_{k}\left[\frac{\omega_{k}}{k} c_{k}+\frac{1}{2 \pi} \int \min \left(k, k_{1}, k_{2}, k_{3}\right), c_{k_{1}}^{*} c_{k_{2}} c_{k_{3}} \delta_{k+k_{1}-k_{2}-k_{3}} d k_{1} d k_{2} d k_{3}\right]=0
$$

which is exactly the super compact equation written in $k$ - space.

The expression in square brackets of (4.4) is the variational derivative of the following Hamiltonian:

$$
H=\int \frac{\omega_{k}}{k}\left|c_{k}\right|^{2} d k+\frac{1}{4 \pi} \int \min \left(k, k_{1}, k_{2}, k_{3}\right) c_{k}^{*} c_{k_{1}}^{*} c_{k_{2}} c_{k_{3}} \delta_{k+k_{1}-k_{2}-k_{3}} d k d k_{1} d k_{2} d k_{3}
$$

Using the following relations between $k$-space and $x$-space,

$$
\begin{array}{rlrl}
k c_{k}^{*} & \Leftrightarrow i \frac{\partial}{\partial x} c^{*}(x), & k c_{k} \Leftrightarrow-i \frac{\partial}{\partial x} c(x), \\
\left|k-k_{2}\right| c_{k}^{*} c_{k_{2}} \Leftrightarrow \hat{k}\left(|c(x)|^{2}\right), & \left(k+k_{1}\right) c_{k} c_{k_{1}} \Leftrightarrow-i \frac{\partial}{\partial x}\left(c(x)^{2}\right),
\end{array}
$$

and definition of $\min \left(k, k_{1}, k_{2}, k_{3}\right)(3.5)$, the new Hamiltonian, whose fourth order is defined by the new coefficient $\tilde{\mathbf{T}}_{\mathbf{k k}_{\mathbf{1}}}^{\mathbf{k}_{\mathbf{2}} \mathbf{k}_{\mathbf{3}}}(4.1$,$) can be written in x$-space:

$$
H=\int c^{*} \hat{V} c d x+\frac{1}{2} \int\left[\frac{i}{4}\left(c^{2} \frac{\partial}{\partial x} c^{* 2}-c^{* 2} \frac{\partial}{\partial x} c^{2}\right)-|c|^{2} \hat{k}\left(|c|^{2}\right)\right] d x
$$


Here, the operator $\hat{V}$ is in k-space so that $V_{k}=\frac{\omega_{k}}{k}$. If one also introduces a bracket similar to the Gardner-Zakharov-Faddeev one (see Zakharov \& Faddev (1971)), then

$$
\partial_{x}^{+} \Leftrightarrow i k \theta_{k}
$$

Then, the equation of motion is the following:

$$
\frac{\partial c}{\partial t}+\partial_{x}^{+} \frac{\delta H}{\delta c^{*}}=0
$$

We introduce the advection velocity

$$
\mathcal{U}=\hat{k}|c|^{2}
$$

and taking a variational derivative, one can write equation (4.8) in the following form:

$$
\frac{\partial c}{\partial t}+i \hat{\omega} c-i \partial_{x}^{+}\left(|c|^{2} \frac{\partial c}{\partial x}\right)=\partial_{x}^{+}(\mathcal{U} c)
$$

Note that $|c|^{2}$ has dimensions of potential. One can recognize two terms in the equation:

- nonlinear wave term: $i \hat{\omega} c-i \partial_{x}^{+}\left(|c|^{2} \frac{\partial c}{\partial x}\right)$

- advection term: $\partial_{x}^{+}(\mathcal{U} c)$.

Along with the usual quantities, such as energy and both momenta, equation (4.10) conserves the action, or the number of waves:

$$
N=\int_{0}^{\infty} \frac{\left|c_{k}\right|^{2}}{k} d k
$$

Equation (4.10) has an exact self-similar solution:

$$
c(x, t)=g\left(t_{0}-t\right)^{\frac{3}{2}} C\left(\frac{x}{g\left(t_{0}-t\right)^{2}}\right) .
$$

It is easy to check that $C(\xi)$ satisfies the following condition:

$$
\frac{3}{2} C-2 \xi \frac{\partial C}{\partial \xi}+i \hat{K}^{\frac{1}{2}} C-i \frac{\partial}{\partial \xi}\left(|C|^{2} \frac{\partial C}{\partial \xi}\right)=\frac{\partial}{\partial \xi}\left(\left(\hat{K}|C|^{2}\right) C\right)
$$

where $C(\xi)$ is a dimensionless function that is analytic in the upper half-plane and $\hat{K}$ is a dimensionless operator.

In $k$-space, this solution (according to (4.4)) has the following form:

$$
c(k, t)=g^{2}\left(t_{0}-t\right)^{\frac{7}{2}} F\left(g k\left(t_{0}-t\right)^{2}\right)
$$

It is easy to check that the dimensionless function $F(\xi)$ satisfies the following equation:

$$
\frac{7}{2} F+2 \xi \frac{\partial F}{\partial \xi}=i \xi^{\frac{1}{2}} F+\frac{i \xi}{2 \pi} \int \min \left(\xi, \xi_{1}, \xi_{2}, \xi_{3}\right) F^{*}\left(\xi_{1}\right) F\left(\xi_{2}\right) F\left(\xi_{3}\right) \delta_{\xi+\xi_{1}-\xi_{2}-\xi_{3}} d \xi_{1} d \xi_{2} d \xi_{3}
$$

This equation may have a solution with singularities, but this has not been studied yet.

\section{Back to $\eta$ and $\psi$}

The physical variables, $\eta_{k}$ and $\psi_{k}$, are hidden in the normal complex variable $c_{k}$ and must be recovered. It is necessary when comparing the theory with experiment.

According to canonical transformation (2.1), $\eta_{k}$ and $\psi_{k}$ are power series of $b_{k}$. On the other hand, using (3.9), the definition of $\tilde{\mathbf{B}}_{\mathbf{k k}_{1}}^{\mathbf{k}_{2} \mathbf{k}_{3}}$ (3.11) with $\tilde{\mathbf{T}}_{\mathbf{k k}_{\mathbf{1}}}^{\mathbf{k}_{2} \mathbf{k}_{\mathbf{3}}}$ (4.1) and relation 
(4.3), they can be easily written as power series of $c_{k}$. The accuracy of the super compact equation provides power series up the third order:

$$
\eta_{k}=\eta_{k}^{(1)}+\eta_{k}^{(2)}+\eta_{k}^{(3)}, \quad \psi_{k}=\psi_{k}^{(1)}+\psi_{k}^{(2)}+\psi_{k}^{(3)} .
$$

The details on recovering the physical quantities $\eta(x, t)$ and $\psi(x, t)$ are given in Dyachenko et al (2015). Here, we present only the linear and second-order terms. All of these terms can be written in $k$-space in a compact form. This is an important property that allows one to recover physical values without multidimensional integrals.

$$
\eta^{(1)}(x)=\frac{1}{\sqrt{2} g^{\frac{1}{4}}}\left(\hat{k}^{-\frac{1}{4}} c(x)+\hat{k}^{-\frac{1}{4}} c(x)^{*}\right), \quad \psi^{(1)}(x)=-i \frac{g^{\frac{1}{4}}}{\sqrt{2}}\left(\hat{k}^{-\frac{3}{4}} c(x)-\hat{k}^{-\frac{3}{4}} c(x)^{*}\right) .
$$

The operators $\hat{k}^{\alpha}$ act in the Fourier space as multiplication by $|k|^{\alpha}$.

$$
\begin{aligned}
\eta^{(2)}(x) & =\frac{\hat{k}}{4 \sqrt{g}}\left[\hat{k}^{-\frac{1}{4}} c(x)-\hat{k}^{-\frac{1}{4}} c(x)^{*}\right]^{2}, \\
\psi^{(2)}(x) & =\frac{i}{2}\left[\hat{k}^{-\frac{1}{4}} c(x)^{*} \hat{k}^{\frac{1}{4}} c(x)^{*}-\hat{k}^{-\frac{1}{4}} c(x) \hat{k}^{\frac{1}{4}} c(x)\right]+ \\
& +\frac{1}{2} \hat{H}\left[\hat{k}^{-\frac{1}{4}} c(x) \hat{k}^{\frac{1}{4}} c(x)^{*}+\hat{k}^{-\frac{1}{4}} c(x)^{*} \hat{k}^{\frac{1}{4}} c(x)\right],
\end{aligned}
$$

Here, $\hat{H}$ is the Hilbert transformation with eigenvalue $i \operatorname{sign}(k)$.

This accuracy (second-order power series) is sufficient to compare numerical data with the data in a flume.

\section{Numerical Simulation}

\subsection{Breather}

The super compact equation (4.10) has a localized breather-type solution:

$$
c(x, t)=C(x-\mathcal{V} t) e^{i\left(k_{0} x-\omega_{0} t\right)} \quad \text { or } \quad c_{k}(t)=e^{i(\Omega+\mathcal{V} k) t} \phi_{k}
$$

where $\phi_{k}$ satisfies the following equation:

$$
\left(\Omega+\mathcal{V} k-\omega_{k}\right) \phi_{k}=\frac{1}{2} \int T_{k k_{1}}^{k_{2} k_{3}} \phi_{k_{1}}^{*} \phi_{k_{2}} \phi_{k_{3}} \delta_{k+k_{1}-k_{2}-k_{3}} d k_{1} d k_{2} d k_{3}
$$

Here, $\mathcal{V}$ is the group velocity and $k_{0}$ and $\omega_{0}$ are the wavenumber and frequency of the carrier wave, respectively. $\Omega$ is close to $\frac{\omega_{0}}{2}$. This solution can be found numerically by the Petviashvili method (see Petviashvili (1976)). A uniform grid is introduced in the periodic domain $x \in[0, L]$. Therefore, the wavenumbers $k$ become discrete, with a stepsize of $\Delta k=\frac{2 \pi}{L}$, and all integrals over $k$ transform to sums over $k$.

$$
\begin{aligned}
& \phi_{k}^{n+1}=\frac{N L_{k}^{n}}{M_{k}}\left[\frac{\sum_{k^{\prime}}\left(\phi_{k^{\prime}}^{n} N L_{k^{\prime}}^{n}\right)}{\sum_{k^{\prime}}\left(\phi_{k^{\prime}}^{n} M_{k^{\prime}} \phi_{k^{\prime}}^{n}\right)}\right]^{-\frac{3}{2}}, \quad M_{k}=\Omega+\mathcal{V} k-\omega_{k}, \\
& N L^{n}=-\frac{\partial^{+}}{\partial x}\left(\left|\phi^{n}\right|^{2} \frac{\partial \phi^{n}}{\partial x}\right)+i \frac{\partial^{+}}{\partial x}\left(\hat{k}\left(\left|\phi^{n}\right|^{2}\right) \phi^{n}\right),
\end{aligned}
$$

Here, $n$ is the number of the iteration. $C(x-\mathcal{V} t)$ has an obvious association with a soliton for the nonlinear Schrödinger equation.

A free surface profile of the breather solution of this equation in the periodic domain $L=10 \mathrm{~km}$ with $k_{0}=\frac{2 \pi}{L} 100$ is shown in Fig.1. The gravity acceleration $g=9.81 \frac{\mathrm{m}}{\sec ^{2}}$. 


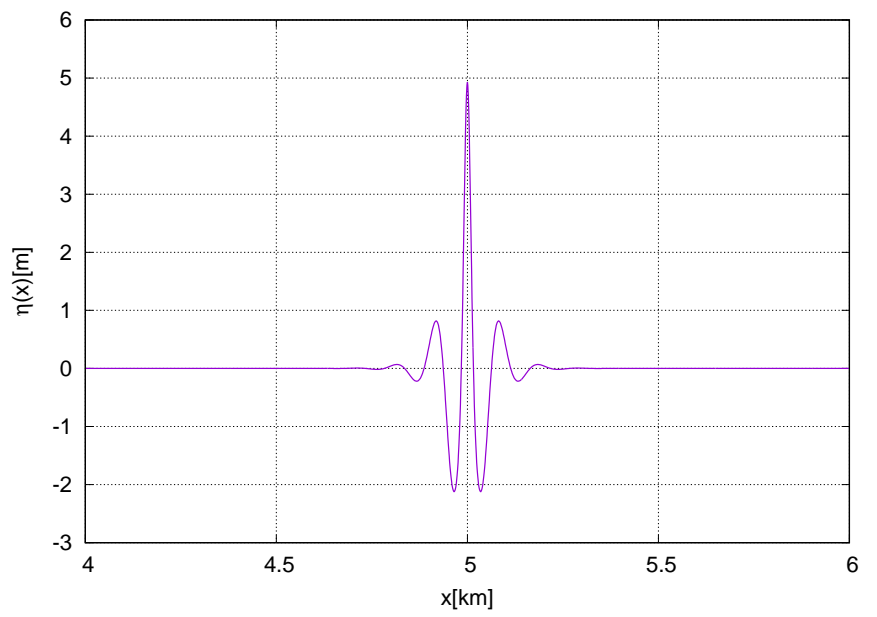

FiguRE 1. Narrow breather with three crests. Free surface profile.

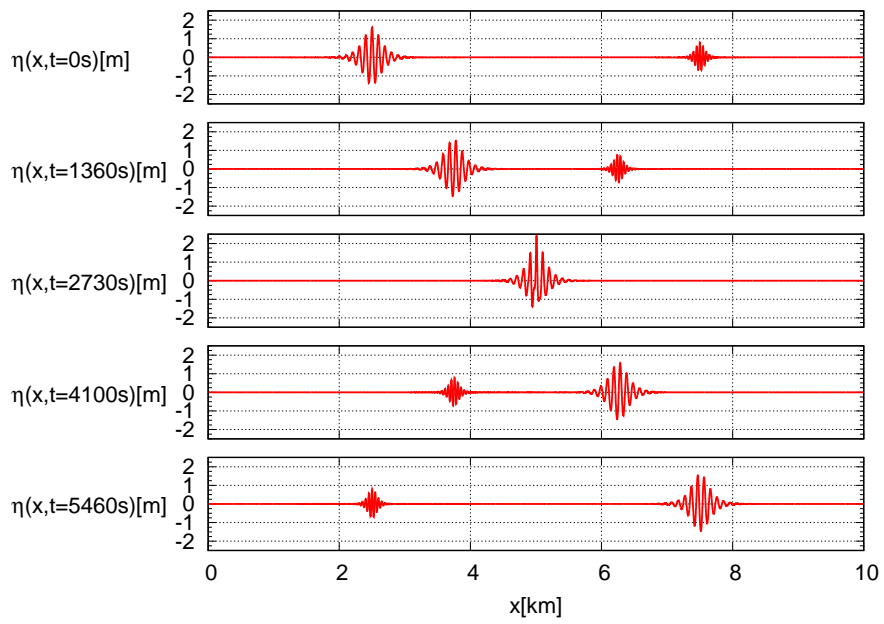

Figure 2. Snapshots of breathers collision

A breather is a very stable structure. A collision of two breathers moving with different velocities (or with $k_{0}=\frac{2 \pi}{L} 100$ and $k_{0}=\frac{2 \pi}{L} 200$ ) is shown in Fig. 2 . An animation of this collision can be viewed in the movie "Collision of 2 breathers" on the JFM site.

\subsection{Modulational instability}

A freak-wave appearing from the slowly modulated Stokes wave of small amplitude $\left(\eta \simeq \eta_{0} \cos \left(k_{0} x-\omega_{k_{0}} t\right)\right)$, with $k_{0}=\frac{2 \pi}{L} 100$ and $\eta_{0} \simeq 1.35 m$, is shown in Fig. 3 :

One can see the beginning of the wave breaking in Fig.4: One can see that the wave is going to break to the right (the right slope of the wave is steeper than the left slope).

The animation of a typical freak wave arising can be found in the two movies, "Freak wave pre-breaking" and "Freak wave pre-breaking zoomed", on the JFM site.

The analytical study of the small-scale instabilities by the "frozen coefficient" method allows one to conclude that the Cauchy problem for the super compact equation is a well-posed problem. 


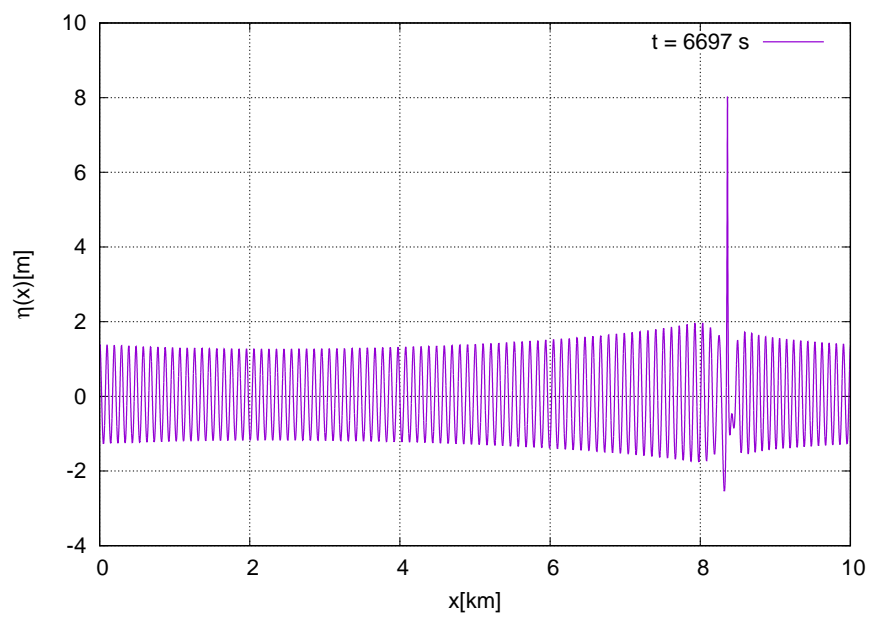

Figure 3. Amplitude of freak-wave in the periodic domain $L=10 \mathrm{~km}$.

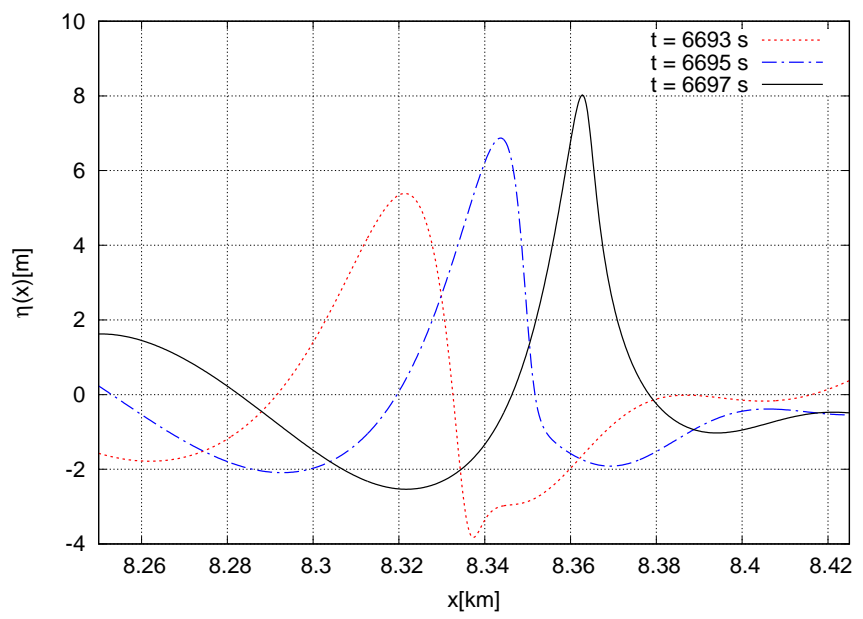

FIGURE 4. Three snapshots showing the beginning of the wave breaking (zoomed near $x=8.34 \mathrm{~km}$

\section{Spatial compact equation}

The simplicity of the super compact equation enables an easy derivation of the spatial version of the equation. The details of this derivation can be found in Dyachenko \& Zakharov (2016). The idea of the derivation is based on the fact that the Fourier image (after transforming equation (4.10) in both space and time) $c_{k \omega}$ is supported on the shadowed area in the vicinity of the dispersion curve, as shown in Fig.5. Note that for unidirectional waves, both $k$ and $\omega$ are positive. This equation (after multiplying by $(\omega+\sqrt{g k}))$ looks like the following:

$$
\begin{aligned}
\left(\omega^{2}-g k\right) c_{k \omega} & =\frac{\left(\omega+\omega_{k}\right) k \theta_{k}}{(2 \pi)^{2}} \int_{k_{i}, \omega_{i}>0} T_{k_{2} k_{3}}^{k k_{1}} c_{k_{1} \omega_{1}}^{*} c_{k_{2} \omega_{2}} c_{k_{3} \omega_{3}} \times \\
& \times \delta_{k+k_{1}-k_{2}-k_{3}} \delta_{\omega+\omega_{1}-\omega_{2}-\omega_{3}} d k_{1} d k_{2} d k_{3} d \omega_{1} d \omega_{2} d \omega_{3}
\end{aligned}
$$

For all $c_{k_{i} \omega_{i}}$, the following relations for their arguments are valid:

$$
\omega_{i}=\sqrt{g k_{i}}+\tilde{\omega}_{n l} .
$$




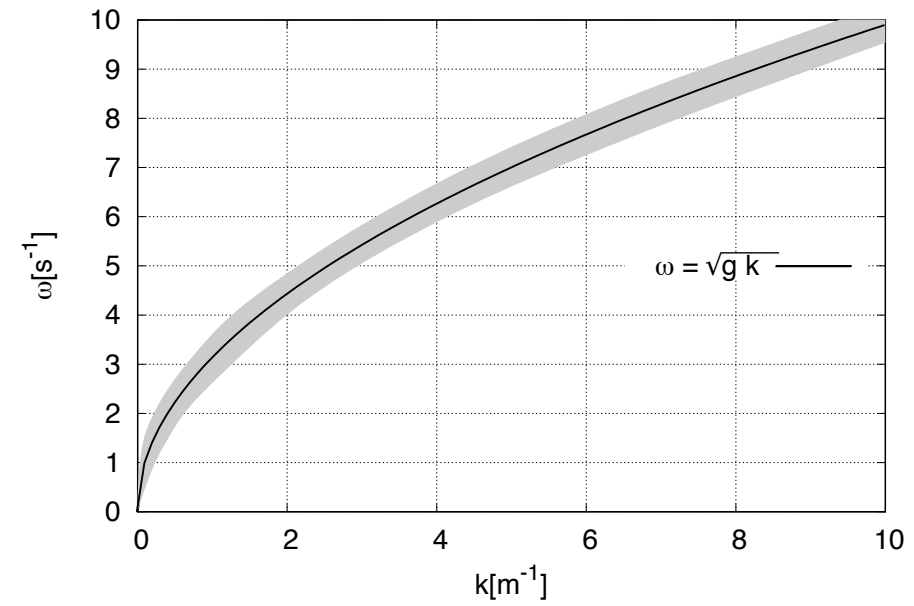

FiguRE 5. Domain (gray) in $k-\omega$ space where all waves evolve

Here, $\tilde{\omega}_{n l}$ is a nonlinear frequency shift, which can be estimated from (7.1) as

$$
\tilde{\omega}_{n l} \sim|c|^{2}
$$

Then, one can replace $T_{k_{2} k_{3}}^{k k_{1}}$ on $T_{\omega_{2}^{2} \omega_{3}^{2}}^{\omega^{2} \omega^{2}}$ and drop all the terms with $\tilde{\omega}_{n l}$. After performing the backward Fourier transformation in $k$-space, the following equation is derived:

$$
\begin{aligned}
\frac{\partial c_{\omega}}{\partial x}-i \frac{\omega^{2}}{g} c_{\omega} & =-\frac{2 \omega^{3} \theta_{\omega}}{g^{3}} \frac{i}{2 \pi} \int T_{\omega_{2}^{2} \omega_{3}^{2}}^{\omega^{2} \omega_{\omega_{1}}^{2}} c_{\omega_{2}} c_{\omega_{3}} \delta_{\omega+\omega_{1}-\omega_{2}-\omega_{3}} d \omega_{1} d \omega_{2} d \omega_{3} \\
T_{\omega_{2}^{2} \omega_{3}^{2}}^{\omega^{2} \omega^{2}} & =\frac{1}{4 \pi} \theta_{\omega} \theta_{\omega_{1}} \theta_{\omega_{2}} \theta_{\omega_{3}} \min \left(\omega^{2}, \omega_{1}^{2}, \omega_{2}^{2}, \omega_{3}^{2}\right) .
\end{aligned}
$$

This is the Hamiltonian spatial equation for water waves with the Hamiltonian

$$
H=\frac{1}{g} \int \frac{1}{\omega}\left|c_{\omega}\right|^{2} d \omega-\frac{1}{2 \pi} \frac{1}{g^{3}} \int T_{\omega_{2}^{2} \omega_{3}^{2}}^{\omega^{2}} c_{\omega}^{2} c_{\omega_{1}}^{*} c_{\omega_{2}} c_{\omega_{3}} \delta_{\omega+\omega_{1}-\omega_{2}-\omega_{3}} d \omega d \omega_{1} d \omega_{2} d \omega_{3} .
$$

The equation of motion $\frac{\partial}{\partial x} c_{\omega}=i \omega^{3} \theta_{\omega} \frac{\delta H}{\delta c_{\omega}^{*}}$ is

$$
\begin{aligned}
\frac{\partial c}{\partial x}+\frac{i}{g} \frac{\partial^{2} c}{\partial t^{2}} & =\frac{\hat{P}^{-}}{2 g^{3}} \frac{\partial^{3}}{\partial t^{3}}\left[\frac{\partial^{2}}{\partial t^{2}}\left(|c|^{2} c\right)+2|c|^{2} \frac{\partial^{2} c}{\partial t^{2}}+c^{2} \frac{\partial^{2} c^{*}}{\partial t^{2}}\right]+ \\
& +\frac{i \hat{P}^{-}}{g^{3}} \frac{\partial^{3}}{\partial t^{3}}\left[\frac{\partial}{\partial t}\left(c \hat{\omega}|c|^{2}\right)+\frac{\partial c}{\partial t} \hat{\omega}|c|^{2}+c \hat{\omega}\left(c^{*} \frac{\partial c}{\partial t}-c \frac{\partial c^{*}}{\partial t}\right)\right]
\end{aligned}
$$

The operator $\hat{P}^{-}$is the projection operator:

$$
\hat{P}^{-}=\frac{1}{2}(1-i \hat{H}), \text { here, } \hat{H} \text { is the Hilbert transformation, }
$$

and it is equal to $\theta_{\omega}$ in Fourier space.

An analytical study of the small-scale instabilities by the "frozen coefficient" method also allows one to conclude that the Cauchy problem for the compact spatial equation is a well-posed problem (although it includes a fifth derivative). 


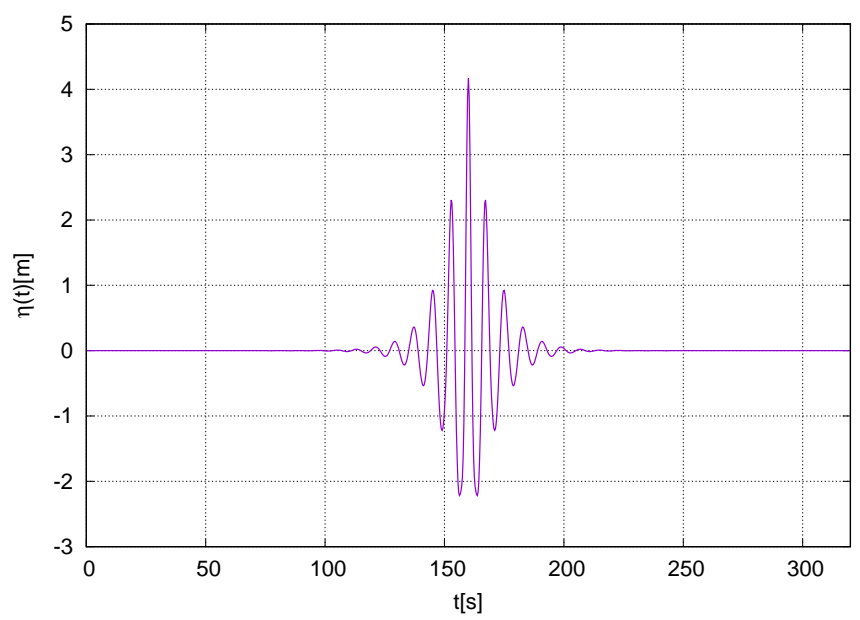

Figure 6. Breather solution. Free surface profile.

\section{Some numerics for spatial equation}

\subsection{Breather}

A breather is the localized solution of the spatial equation of the following type:

$$
c(x, t)=C\left(t-\frac{x}{\mathcal{V}}\right) e^{i\left(k_{0} x-\omega_{0} t\right)}
$$

Fourier transforming over time, one can obtain:

$$
c_{\omega}(x)=\frac{1}{\sqrt{2 \pi}} \int C\left(t-\frac{x}{\mathcal{V}}\right) e^{i k_{0} x-i\left(\omega_{0}-\omega\right) t} d t=\frac{1}{\sqrt{2 \pi}} \int C(\xi) e^{-i\left(\omega_{0}-\omega\right) \xi} e^{i k_{0} x-i\left(\omega_{0}-\omega\right) \frac{x}{\mathcal{V}}} d \xi,
$$

or

$$
c_{\omega}(x)=\phi_{\omega} e^{i\left(\mathcal{K}+\frac{\omega}{\nu}\right) x}
$$

Here, $\mathcal{K}=k_{0}-\frac{\omega_{0}}{\mathcal{V}}$ is close to $-\frac{\omega_{0}^{2}}{g}$, and $\phi_{\omega}$ satisfies the following equation:

$$
\left(\mathcal{K}+\frac{\omega}{\mathcal{V}}-\frac{\omega^{2}}{g}\right) \phi_{\omega}=-\frac{2 \omega^{3} \theta_{\omega}}{g^{3}} \frac{1}{2 \pi} \int T_{\omega^{2} \omega_{1}^{2}}^{\omega_{2}^{2} \omega_{\omega_{1}}^{2}} \phi_{\omega_{2}} \phi_{\omega_{3}} \delta_{\omega+\omega_{1}-\omega_{2}-\omega_{3}} d \omega_{1} d \omega_{2} d \omega_{3}
$$

This can be found by use of the iterative Petviashvili method ( $n$ is the number of the iteration). A uniform grid is introduced in the periodic domain $t \in[0, T]$. Therefore, the frequencies $\omega$ become discrete, with a stepsize of $\Delta \omega=\frac{2 \pi}{T}$, and all integrals over $\omega$ transform to sums over $\omega$.

$$
\begin{aligned}
\phi_{\omega}^{n+1} & =\frac{N L_{\omega}^{n}}{M_{\omega}}\left[\frac{\sum_{\omega^{\prime}}\left(\phi_{\omega^{\prime}}^{n} N L_{\omega^{\prime}}^{n}\right)}{\sum_{\omega^{\prime}}\left(\phi_{\omega^{\prime}}^{n} M_{\omega^{\prime}} \phi_{\omega^{\prime}}^{n}\right)}\right]^{-\frac{3}{2}}, \quad M_{\omega}=\mathcal{K}+\frac{\omega}{\mathcal{V}}-\frac{\omega^{2}}{g} \\
N L^{n} & =\frac{-i \hat{P}^{-}}{2 g^{3}} \frac{\partial^{3}}{\partial t^{3}}\left[\frac{\partial^{2}}{\partial t^{2}}\left(\left|\phi^{n}\right|^{2} \phi^{n}\right)+2\left|\phi^{n}\right|^{2} \frac{\partial^{2} \phi^{n}}{\partial t^{2}}+\frac{\partial^{2} \phi^{n *}}{\partial t^{2}} \phi^{n 2}\right]+ \\
& +\frac{\hat{P}^{-}}{g^{3}} \frac{\partial^{3}}{\partial t^{3}}\left[\frac{\partial}{\partial t}\left(\phi^{n} \hat{\omega}\left|\phi^{n}\right|^{2}\right)+\frac{\partial \phi^{n}}{\partial t} \hat{\omega}\left|\phi^{n}\right|^{2}+\phi^{n} \hat{\omega}\left(\frac{\partial \phi^{n}}{\partial t} \phi^{n *}-\phi^{n} \frac{\partial \phi^{n *}}{\partial t}\right)\right]
\end{aligned}
$$

A free surface profile of the breather solution of this equation, in the periodic domain $T=320 s$, with $\omega_{0}=0.78\left[s^{-1}\right]$ and $\mathcal{K}=-6.428 \cdot 10^{-2}$, is shown in Fig.6. The Fourier harmonics $\left(\left|c_{\omega}\right|\right)$ of the breather solution in a logarithmic scale are shown in Fig.7. 


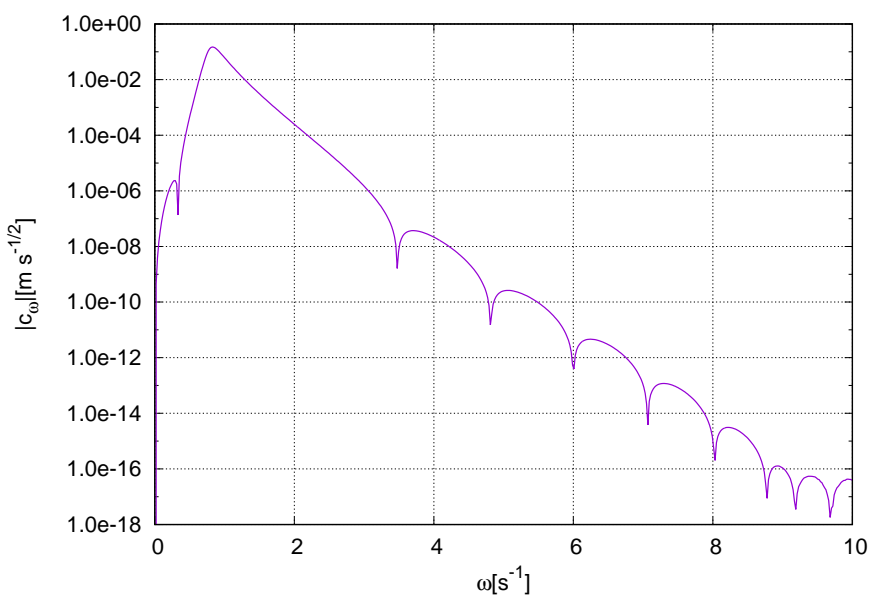

Figure 7 . The Fourier harmonics $\left(\left|c_{\omega}\right|\right)$ of the breather solution.

An animation of breather generation in a "digital" flume can be found in the movie "Breathers in a flume" on the JFM site.

\subsection{Modulational instability}

In the spatial equation, the modulational instability of a monochromatic wave also occurs. The monochromatic wave

$$
c(x, t)=c_{0} e^{i k_{0} x-i \omega_{0} t}, \text { or } c(x, \omega)=\sqrt{2 \pi} c_{0} e^{i k_{0} x} \delta\left(\omega-\omega_{0}\right)
$$

is the simplest solution of (7.3) and (7.2). Substituting (8.5) into (7.2) yields the following relation:

$$
k_{0}=\frac{\omega_{0}^{2}}{g}-\frac{2 \omega_{0}^{5}}{g^{3}}\left|c_{0}\right|^{2},
$$

$\left(-\frac{2 \omega_{0}^{5}}{g^{3}}\left|c_{0}\right|^{2}\right.$ could be called a "nonlinear wavelength shift"). The perturbed solution has the following form:

$$
c(x, \omega)=\sqrt{2 \pi}\left(c_{0} \delta\left(\omega-\omega_{0}\right)+c_{+}(x) \delta\left(\omega-\omega_{+}\right) e^{i k_{+} x}+c_{-}(x) \delta\left(\omega-\omega_{-}\right) e^{i k_{-} x}\right) e^{i k_{0} x}(8.7)
$$

Here, $\omega_{ \pm}=\omega_{0} \pm \omega$ and $k_{+}=-k_{-}$with the following condition:

$$
\left|c_{+}\right|,\left|c_{-}\right|<<\left|c_{0}\right| \text {. }
$$

Substituting (8.7) into (7.2), one can obtain the sum of two independent equations:

$$
\begin{aligned}
& {\left[\frac{\partial c_{+}}{\partial x}+i\left(k_{0}+k_{+}\right) c_{+}-\frac{i}{g} \omega_{+}^{2} c_{+}+\frac{4 i \omega_{+}^{3}}{g^{3}} T_{\omega_{+}^{2} \omega_{0}^{2}}^{\omega_{2}^{2} \omega_{0}^{2}}\left|c_{0}\right|^{2} c_{+}+\frac{2 i \omega_{+}^{3}}{g^{3}} T_{\omega_{0}^{2} \omega_{0}^{2}}^{\omega^{2} \omega_{-}^{2}} c_{0}^{2} c_{-}^{*}\right] e^{i k_{+} x}+} \\
& +\left[\frac{\partial c_{-}}{\partial x}+i\left(k_{0}+k_{-}\right) c_{-}-\frac{i}{g} \omega_{-}^{2} c_{-}+\frac{4 i \omega_{-}^{3}}{g^{3}} T_{\omega_{-}^{2} \omega_{0}^{2}}^{\omega^{2} \omega_{0}^{2}}\left|c_{0}\right|^{2} c_{-}+\frac{2 i \omega_{-}^{3}}{g^{3}} T_{\omega_{0}^{2} \omega_{0}^{2}}^{\omega^{2} \omega^{2}} c_{0}^{2} c_{+}^{*}\right] e^{i k_{-} x}=0
\end{aligned}
$$

Expressions for $T_{\omega_{+}^{2} \omega_{0}^{2}}^{\omega_{2}^{2} \omega_{2}^{2}}, T_{\omega_{0}^{2} \omega_{0}^{2}}^{\omega^{2} \omega_{-}^{2}}$ and $T_{\omega_{-}^{2} \omega_{0}^{2}}^{\omega^{2} \omega^{2}}$ can be easily obtained from (4.1). Suppose that $c_{ \pm}$grow according to

$$
c_{ \pm} \Rightarrow c_{ \pm} e^{\gamma_{\omega} x}
$$

Then, we can obtain the formula for $\gamma_{\omega}$ given by a tenth-degree polynomial. By introducing steepness for the monochromatic wave $\eta(x)=\eta_{0} \cos k_{0} x$ as $\mu=\eta_{0} k_{0}$, one can 


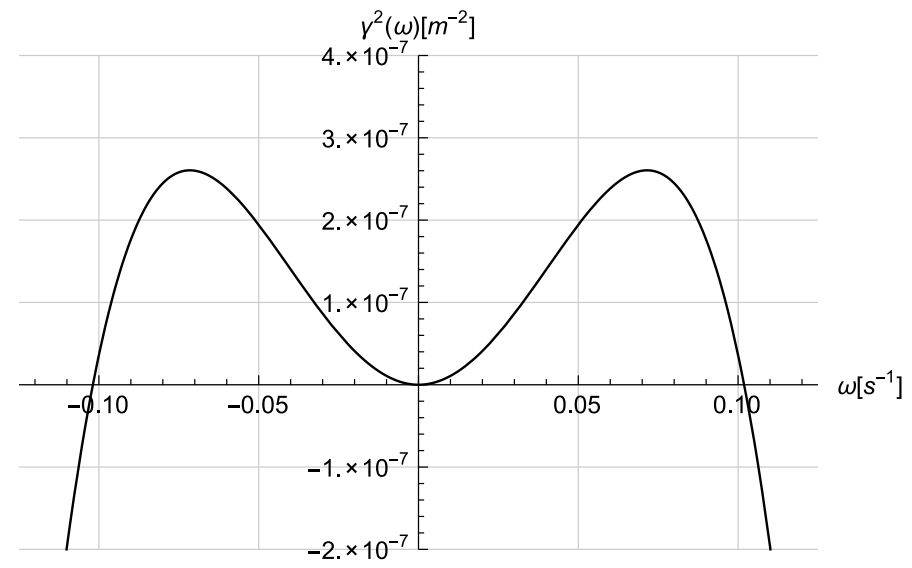

FiguRE 8 . The growth rate squared $\gamma^{2}(\omega)$ of the perturbation $c_{ \pm}$of the monochromatic wave solution of the spatial equation (7.2). Here, $\omega_{0}=0.78\left[\mathrm{~s}^{-1}\right]$, and steepness of the carrier wave $\mu \approx 0.1$.

easily find (see Section 5) that in terms of $c(x)=c_{0} e^{i k_{0} x}$,

$$
\mu=\frac{\sqrt{2}\left|c_{0}\right| \omega_{0}^{\frac{3}{2}}}{g}
$$

The growth rate squared $\gamma^{2}(\omega)$ for $\omega_{0}=0.78\left[s^{-1}\right]$, and the steepness of the carrier wave $\mu=0.1$ is shown in Fig. 8. Perturbations whose frequencies $\omega$ are such that $\gamma^{2}(\omega)>0$ are unstable, and they grow as $c_{ \pm} \sim e^{\gamma(\omega) x}$. Perturbations whose frequencies $\omega$ are such that $\gamma^{2}(\omega)<0$ are stable; therefore, they only change phase $c_{ \pm} \sim e^{i \sqrt{-\gamma^{2}(\omega)} x}$.

\section{Conclusion}

We derived and discussed a new compact and elegant form of the Hamiltonian and equation for the gravity waves at the surface of deep water. Starting with the classical canonical variables $\left(\eta_{k}, \psi_{k}\right)$, the equation was derived in four steps.

First, the normal complex variable $a_{k}$ was introduced in Section 2.

Second, a canonical transformation was applied to eliminate the nonresonant terms (third and fourth order) in the Hamiltonian. As the result, we obtained the Zakharov equation and observed that the four-wave coefficient has a remarkable property in the 1-D case:

$$
T_{k k_{1}}^{k_{2} k_{3}} \equiv 0 \quad \text { if the product } k k_{1} k_{2} k_{3} \leqslant 0 .
$$

The fact that $T_{k k_{1}}^{k_{2} k_{3}}$ is zero on the resonant manifold is just part of the above.

Third, this property allowed us to simplify $T_{k k_{1}}^{k_{2} k_{3}}$ by applying another canonical transformation. As a result, the compact equation with an explicit form for $T_{k k_{1}}^{k_{2} k_{3}}$ in $x$-space was derived (see (3.13).

Fourth, we derived probably the simplest form of the Hamiltonian and equation for 1-D water waves, where the order of the differential equation was reduced from 3 to 2 . We call this the super compact equation.

The equation allows one to obtain a spatial version of the water wave equation that is suitable for the simulation of a laboratory experiment whereby the free surface is governed by wavemakers. Cauchy problems for both temporal and spatial equations are well-posed problems. 
Thus,

- the Hamiltonian of the super compact equation, in both $k$-space (4.5) and $x$-space (4.6), is very simple;

- the equation itself is very straightforward, consisting of only two terms - nonlinear waves and advection;

- advection is obviously responsible for wave-breaking, and the super compact equation can describe the pre-breaking wave; and

- it can be easily implemented for numerical simulations.

The equation can be generalized for "almost" 2-D waves, just as the KdV is generalized to the Kadomtsev-Petviashvili equation:

$$
\begin{aligned}
H & =\int c^{*} \hat{V} c d x d y+ \\
& +\frac{1}{2} \int\left[\frac{i}{4}\left(c^{2} \frac{\partial}{\partial x} c^{* 2}-c^{* 2} \frac{\partial}{\partial x} c^{2}\right)-|c|^{2} \hat{k}_{x}\left(|c|^{2}\right)\right] d x d y
\end{aligned}
$$

Here, the operator $\hat{V}$ in k-space is $V_{\vec{k}}=\frac{\omega_{\vec{k}}}{k_{x}}$.

\section{Acknowledgments}

This work was supported by Grant "Wave turbulence: theory, numerical simulation, experiment" \#14-22-00174 of the Russian Science Foundation.

\section{REFERENCES}

Dysthe,K.B. 1979 Note on a modification to the nonlinear Schrödinger equation for application to deep water waves, Proc. Roy. Soc. London, Ser. A, 369, 105-114

Zakharov, V.E. 1968 Stability of periodic waves of finite amplitude on the surface of a deep fluid, Journal of Applied Mechanics and Technical Physics 9(2), 190-194

Korotkevich, A.O., Pushkarev, A.N., Resio, D. \& Zakharov, V. 2008 Numerical verification of the weak turbulent model for swell evolution, European Journal of Mechanics - B/Fluids 27(4), 361-387

Crawford,D.E., Yuen,H.G. AND Saffman,P.G. 1980 Evolution of a random inhomogeneous field of nonlinear deep-water gravity waves, Wave Motion, 2(1), 1-16

Debnath, L. 1994 Nonlinear water waves, Academic press Inc.

Annenkov, S.Y. And Shrira, V.I. 2011 Evolution of Wave Turbulence under "Gusty" Forcing, Phys. Rev. Lett., 107, 114502.

Annenkov, S.Y. AND ShriRA, V.I. 2013 Large-time evolution of statistical moments of windwave fields, Journal of Fluid Mechanics, 726, 517-546.

Dyachenko, A.I. And V.E.Zakharov, V.E. 1994 Is free-surface hydrodynamics an integrable system? Phys. Lett. A, 190, 144-148.

Dyachenko, A.I., Zakharov, V.E. 2012 A dynamic equation for water waves in one horizontal dimension, European Journal of Mechanics - B/Fluids 32, 17-21

Fedele, F. And Dutykh, D. 2012a Special solutions to a compact equation for deep-water gravity waves, Journal of Fluid Mechanics 712, 646-660

Fedele, F. AND Dutykh, D. 2012b Solitary wave interaction in a compact equation for deepwater gravity waves, JETP Letters 95(12), 622-625

Zakharov, V.E. And FAdDEV, L.D. $1971 \mathrm{KdV}$ equation: A completely integrable Hamiltonian system, Functional Analysis and Its Applications 5(4), 18-27

Dyachenko,A.I., Kachulin, D.I., Zakharov, V.E. 2013 Collisions of two breathers at the surface of deep water, Nat. Hazards Earth Syst. Sci. 13, 1-6

Dyachenko A.I., Kachulin D.I. And Zakharov V.E. 2014 Freak waves at the surface of deep water, Journal of Physics: Conference Series 510, 012050

Fedele, F. 2014a On certain properties of the compact Zakharov equation, Journal of Fluid Mechanics $\mathbf{7 4 8}, 692-711$ 
Fedele, F. 2014b On the persistence of breathers at deep water, JETP Letters 98(9), 523-527

Dyachenko, A.I., Kachulin, D.I., Zakharov, V.E. 2015 Freak-waves: Compact Equation vs Fully Nonlinear One, "Extreme Ocean Waves" 2nd ed., eds. E. Pelinovsky and C. Kharif. Springer, 23-44

Petviashvili, V.I. 1976 Equation for an extraordinary soliton, Sov. J. Plasma Phys. 2, 257258.

Dyachenko,A.I., Kachulin, D.I., Zakharov, V.E. 2015a Evolution of one-dimensional winddriven sea spectra, Pis'ma $v$ ZhETF 102(8), 577-581

Dyachenko,A.I., Kachulin, D.I., Zakharov, V.E. 2015b Probability Distribution Functions of freak-waves: nonlinear vs linear model, Stud. in Appl. Math in press

Dyachenko, A.I., Kachulin, D.I., Zakharov, V.E. 2013a On the Nonintegrability of the Free Surface Hydrodynamics, JETP. Lett. 98(1), 43-47,

Dyachenko, A.I. And Zakharov, V.E. 2016 Spatial Equation for Water Waves, JETP. Lett. 103(3), 181-184, Pis'ma v ZhETF. 103(3), 200-203

Zakharov, V.E. 1998 Nonlinear waves and wave turbulence, Amer. Math. Soc. Transl. Series 2 182, 167-197

Zakharov, V.E. 1999 Statistical theory of gravity and capillary waves on the surface of a finite-depth fluid, European Journal of Mechanics - B/Fluids 18(3), 327-344

KRASITSKII,V.P. 1990 Canonical transformation in a theory of weakly nonlinear waves with a nondecay dispersion law, Sov. Phys. JETP 71,921

Zakharov V.E., Lvov V.S. And Falkovich G. 1992 Kolmogorov Spectra of Turbulence I, Springer-Verlag

Dyachenko A.I., Lvov Y.V. And Zakharov V.E. 1995 Physica D , 87, 233-261. 